\title{
Um olhar físico sobre a teoria musical
}

A physical look at musical theory

\author{
Joseclécio Dutra Dantas ${ }^{* 1}$, Sergio da Silva Cruz ${ }^{1}$ \\ ${ }^{1}$ Universidade Federal de Campina Grande, Unidade Acadêmica de Física e Matemática, Cuité, PB, Brasil
}

Recebido em 11 de Abril, 2018. Revisado em 23 de Maio, 2018. Aceito em 12 de Junho, 2018.

\begin{abstract}
Apresentamos uma discussão sobre diversos conceitos físicos presentes no contexto da teoria musical. Tratamos de fenômenos físicos importantes na produção e combinação de sons musicais. A partir de elementos próprios tanto da linguagem musical quanto da linguagem física, evidenciamos relações entre as duas formas de representar os fenômenos sonoros musicais. Traçamos paralelos entre as formas de representar as diversas características do som e suas combinações rítmicas, melódicas e harmônicas.
\end{abstract}

Palavras-chave: Sons musicais, Combinações sonoras, Representação do som.

\begin{abstract}
We present a discussion of several physical concepts present in the context of musical theory. We deal with important physical phenomena in the production and combination of musical sounds. From the elements of both musical language and physical language, we show relationships between the two forms of representing musical sound phenomena. We draw parallels between the ways of representing the various characteristics of sound and their rhythmic, melodic and harmonic combinations.
\end{abstract}

Keywords: Musical sounds, Sound combinations, Sound representation.

\section{Introdução}

Cotidianamente percebemos que fenômenos comuns, naturais ou controlados, envolvendo corpos em vibração têm como uma de suas consequências a produção de ondas sonoras - ondas mecânicas longitudinais, com frequências aproximadamente entre os limites $20 \mathrm{~Hz}$ e $20 \mathrm{kHz}$, que nos causam a sensação da audição [1] 3]. Esses sons são dos mais variados tipos, cujas características dependem inicialmente da fonte sonora. A parte da Física que se dedica ao estudo dos fenômenos sonoros, como a emissão, a interferência, a reflexão, a difração e demais fenômenos é a Acústica [4], mas isso também é preocupação da Música enquanto arte. Em linguagem física, é possível descrever o fenômeno ondulatório responsável pela emissão sonora, bem como representá-lo, considerando suas qualidades tais como intensidade, frequência e timbre, entre outras; tal representação se dá por meio de expressões matemáticas, tabelas e gráficos [5]. A notação musical também se preocupa em indicar todas essas características através de uma representação gráfica de leitura mais rápida a partitura [6 8]. O que talvez não seja muito evidente é que ambas as linguagens, física e musical, em muitos casos representam os mesmos fenômenos sonoros de maneiras diferentes. O objetivo deste trabalho é, portanto, evidenciar as relações entre a forma de representar o som própria da escrita musical e a representação matemática dos fenômenos físicos ocorridos na produção e nas diversas possibilidades de combinar os sons. Nesse sentido, a

*Endereço de correspondência: jddantas@ufcg.edu.br
Seção 2 traz discussões preliminares sobre as qualidades do som e como elas são entendidas e representadas a partir dos pontos de vista da Física e da Música. Na Seção 3 tratamos de entender, também a partir dos dois pontos de vista citados, como se dá a estruturação do rítmo. As relações de frequências, as combinações de sons sucessivos, a definição das notas e o estudo das estruturas melódicas são assunto da Seção 4 . A Seção 5 trata das combinações harmônicas dos sons, ou seja, das diversas possibilidades de superpor sons simultaneamente e dos padrões harmônicos resultantes. Os comentários finais estão na Seção 6 .

\section{Em busca da representação do som}

O som tem qualidades bastante interessantes que lhe permitem ser classificado como forte ou fraco, grave ou agudo, e demais características que se configuram como elementos importantes a serem estudados no campo da Física e ou da Música. De maneiras distintas nestas duas áreas, o som carece de representação. As composições musicais, em muitas situações, precisam ser registradas em uma simbologia própria da Música. Os fenômenos sonoros, na qualidade de fenômenos físicos, são também representáveis em uma linguagem própria da Física. Uma linguagem que seja eficaz na representação do som deve ser capaz de indicar suas características na totalidade exigida pelo contexto. Quem quer que seja que se dedique a ouvir atentamente um trecho de uma obra musical de qualquer natureza perceberá uma sucessão de sons 
distinguíveis pelas durações, pelas intensidades, pelos timbres, pelas alturas relativas. Quem se dedica ao estudo do som, no contexto da Física ou da Música, deve ser capaz, também, de observar tais características a partir da representação.

No que diz respeito especificamente à Música Ocidental, a notação utilizada é denominada notação diastemática, em que os sons são representados graficamente de tal maneira que seja possível mensurar os intervalos de frequências indicando diferentes notas musicais. A notação musical ocidental tem origem nos neumas ("sinais" em grego), surgidos no século IX, sendo inicialmente símbolos colocados acima do texto para indicar as relações melódicas entre as sílabas musicais. Os neumas continham informações gerais sobre o contorno melódico, mas não indicavam as relações intervalares entre as notas, ou seja, não indicavam tons exatos, embora, com o passar do tempo, tenham chegado a representações mais aproximadas [9]. Não era possível, portanto, que a notação desse conta de representar todos os elementos de uma música sem que se valesse da tradição oral, o que significa que não seria possível que um músico tocasse uma música pela primeira vez simplesmente olhando para a escrita musical, sem ouvi-la antes. Isso é possível com a notação atual que, resultado de uma longa história de contribuições no sentido de melhor adaptar a cada época a forma de representar o som, da mesma forma que a linguagem matemática utilizada pela Física, consegue representar com exatidão as tonalidades, as durações, as intensidades e suas variações. É da notação atual que tratamos.

\subsection{O tempo}

Tendo em vista a necessidade da escrita musical de representar sons relativamente curtos e longos, nossas primeiras considerações serão sobre a duração das notas musicais. Nesse sentido, a grandeza física tempo surge quantificada na forma das figuras musicais [10, que representam padrões de durações tanto do som (as notas) quanto do silêncio (as pausas), conforme ilustra a Figura 1 .

Percebamos que as figuras musicais representam unidades de tempo que, diferentemente das unidades segundo, minuto, hora, dia, ano, não têm, necessariamente, valor fixo, embora as relações entre as diferentes figuras sejam dadas por razões fixas. A figura nomeada por semibreve é a de maior valor temporal. As figuras seguintes, mínima, seminima, colcheia, semicolcheia, fusa, e semifusa, são definidas a partir das frações da semibreve que elas representam. Um quadro comparativo das durações relativas das figuras musicais pode ser visto na Figura 2 Podemos observar que a duração de uma nota representada pela figura semibreve é equivalente à duração de trinta e duas fusas ou sessenta e quatro semifusas, por exemplo, desde que considerado o mesmo padrão de marcação do tempo.

\begin{tabular}{l|c|c|c} 
Nome & Som & Silêncio & Duração \\
\hline Semibreve & 0 & - & 1 \\
\hline Mínima & d & - & $1 / 2$ \\
\hline Semínima & & & $1 / 4$ \\
\hline Colcheia & & & 1 \\
\hline Semicolcheia & & $y$ & $1 / 8$ \\
\hline Fusa & & $y$ & $1 / 16$ \\
\hline Semifusa & & $y$ & $1 / 32$ \\
\hline
\end{tabular}

Figura 1: Figuras musicais representativas das durações temporais relativas.

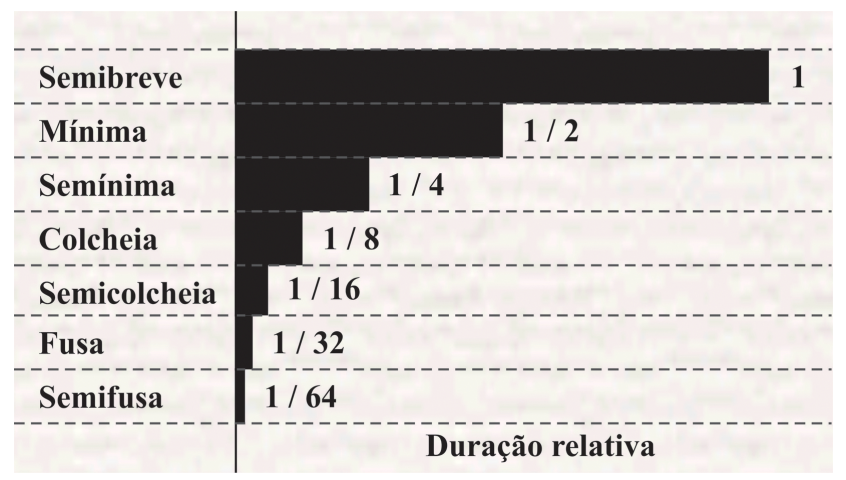

Figura 2: Durações relativas das figuras musicais.

\subsection{A frequência}

Outra qualidade do som de grande importância é a frequência. Esta grandeza é responsável pela classificação dos sons como agudos ou graves. As ondas sonoras de maiores frequências são percebidas como mais agudas do que as ondas sonoras de frequências menores. Na Figura 3, vemos a representação de três ondas senoidais de mesma amplitude e frequências distintas. Ondas sonoras que pudessem, de maneira bastante simplificada, ser representadas por tais funções, seriam sons de alturas diferentes: a curva vermelha representaria o som mais grave; a linha verde representaria o som mais agudo, tendo em vista a ordem de crescimento de frequência ser vermelho-azul-verde. No contexto da simbologia musical, a representação desta qualidade é feita escrevendo-se as figuras de valores apresentadas anteriormente em uma estrutura formada por um conjunto de cinco linhas e quatro espaços, denominada pentagrama [10], conforme ilustra a Figura 4 A lógica é que, pensando verticalmente, notas escritas mais acima representam frequências maiores, e notas escritas mais abaixo representam frequências menores. Assim sendo, da esquerda para a direita, as figuras representam sons cada vez mais agudos. O uso de linhas e espaços suplementares, que não ficam visíveis na escrita musical, se dá pela insuficiência das linhas e espaços do 


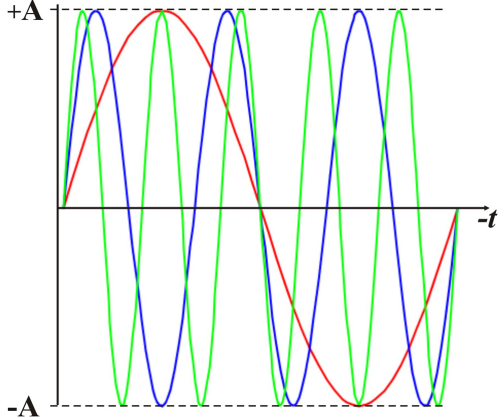

Figura 3: Ondas senoidais de diferentes frequências.

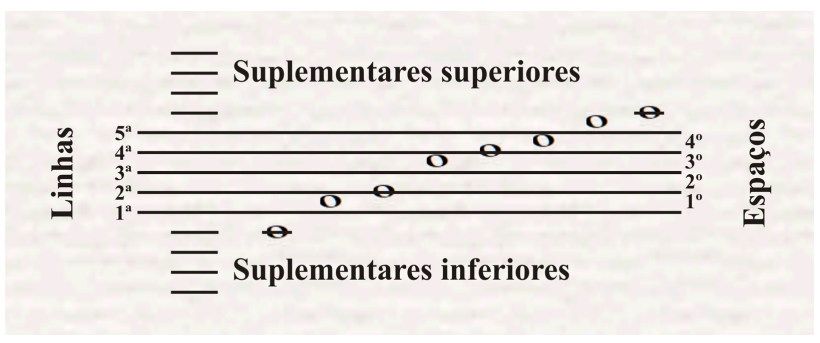

Figura 4: Indicações de frequências no pentagrama.

pentagrama em representar todas as frequências que são emitidas durante a execução de uma peça musical.

As notas escritas no pentagrama da Figura 4 no entanto, ainda não são nomeadas. A nomeação das notas só é possível a partir do uso de um símbolo denominado clave. Existem diferentes tipos de clave; sua função é nomear uma linha, de maneira que, conhecida a sequência já estabelecida das notas musicais - Dó, Ré, Mi, Fá, Sol, $L a ́$, $S i$-, todas as linhas e espaços do pentagrama passam a representar frequências específicas 11]. A Figura 5apresenta três claves mais conhecidas. A primeira, a clave de Sol, é escrita na segunda linha; e isto significa que o sinal

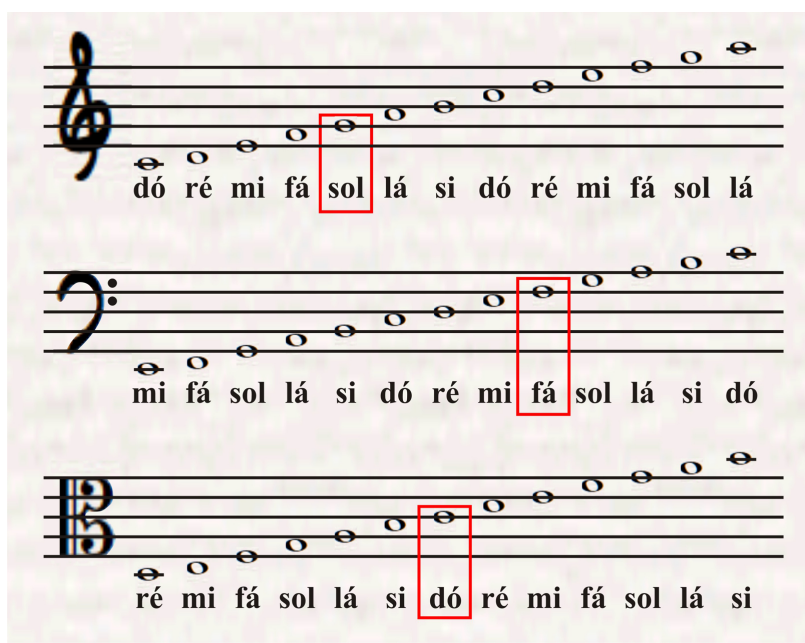

Figura 5: Exemplos de claves: clave de Sol na segunda linha, clave de Fá na quarta lina, clave de Dó da terceira linha, respectivamente. escrito sobre esta linha representa a nota Sol, ou seja, uma onda sonora cuja frequência fundamental é definida para o instrumento musical em questão. Na partitura de um instrumento como o piano, por exemplo, a segunda linha nomeada pela clave de Sol indica uma frequência fundamental de 392, $00 \mathrm{~Hz}$. A segunda clave na Figura 5 é a clave de Fá escrita na quarta linha, que representa a nota Fá. Ainda considerando o piano como instrumento de referência, esta linha representa uma onda sonora cuja frequência fundamental é de 174,61 Hz 12,13. Citamos o piano por ser um instrumento cuja partitura representa as notas reais. Há, no entanto, instrumentos, como o saxofone e o clarinete, que são instrumentos de transposição, ou seja, cujas partituras não indicam as frequências reais, mas uma transposição delas. Há ainda outros tipos de claves que são utilizadas de acordo com a conveniência. A terceira clave na Figura 5 por exemplo, é a clave de Dó na terceira linha, indicando que a nota escrita nesta linha é um Dó, de frequência 261,63 Hz 12,13.

\subsection{A intensidade}

Outra característica importante das ondas sonoras é a intensidade, que permite classificar os sons como mais fortes e mais fracos. Esta grandeza é proporcional ao quadrado da amplitude $A$ da onda [3], da forma

$$
I \propto A^{2}
$$

A Figura 6 ilustra ondas de amplitudes diferentes. A amplitude da onda representada pela curva azul é o dobro da amplitude da onda representada pela curva vermelha. Portanto, a onda da curva azul tem uma intensidade que é quatro vezes a intensidade da onda da curva vermelha.

O som da onda da curva vermelha é, portanto, quatro vezes mais forte. No contexto musical, as relações entre sons mais fortes e mais fracos têm papel importante no que é denominado dinâmica musical. Há também uma simbologia para indicar tais relações. Sinais como $p p, p, m p, m f, f$ e $f f$ indicam, em italiano, sons, respectivamente, pianíssimo (muito fraco), piano (fraco), mezzo piano (moderadamente fraco), mezzo forte (moderadamente intenso), forte (intenso) e fortíssimo (muito intenso).

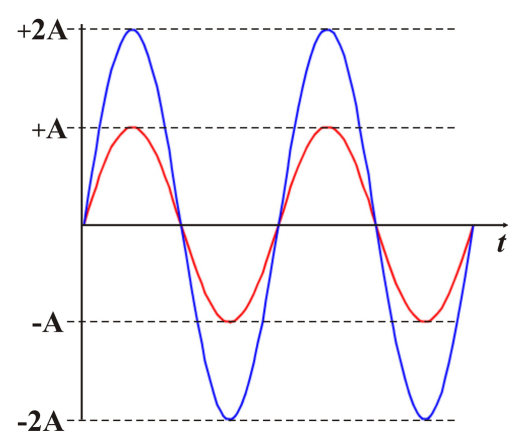

Figura 6: Ondas senoidais de amplitudes diferentes. 
Há ainda sinais que servem para indicar variações na intensidade; é o caso do crescendo < e do decrescendo (ou diminuendo) $>$. A forma como a intensidade varia durante o crescendo pode ser dada por uma função que cresce linearmente no tempo, da forma

$$
I(t)=I_{0} t
$$

Nesse caso, supondo que a onda sonora possa ser representada por uma única função senoidal, esta teria uma amplitude $A(t)=A_{0} t^{1 / 2}$, onde $A_{0} \propto \sqrt{I_{0}}$, sendo dada por

$$
y_{c}=A(t) \sin (2 \pi f t),
$$

onde $f$ indica a frequência da onda. Ilustrações da intensidade e da amplitude durante o crescendo podem ser vistas, respectivamente, nas Figuras 7] e 8. Na Figura 8. as envoltórias azul e verde indicam, respectivamente, $A(t)$ e $-A(t)$.

\subsection{O timbre}

Um outro aspecto que devemos abordar é a possibilidade de distinção entre sons emitidos por diferentes instrumentos. Os sons produzidos por um clarinete e por um trombone, por exemplo, são claramente diferentes, mesmo que estes emitam ondas sonoras com a mesma frequência fundamental. Isso acontece porque a geometria do instrumento e o material do qual é feito determinam as várias frequências que estarão presentes, e como estarão presentes, na emissão de cada som. A qualidade do som

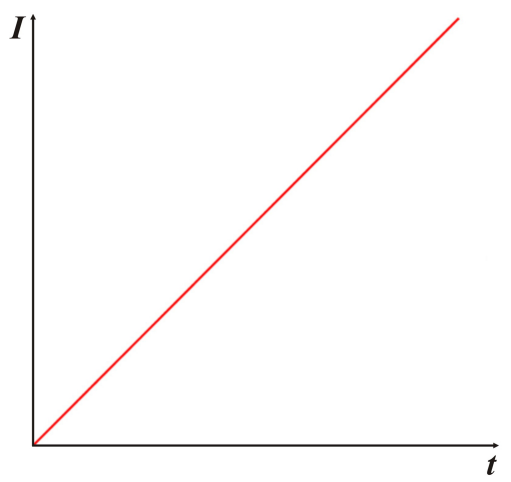

Figura 7: Intensidade sonora durante o crescendo.

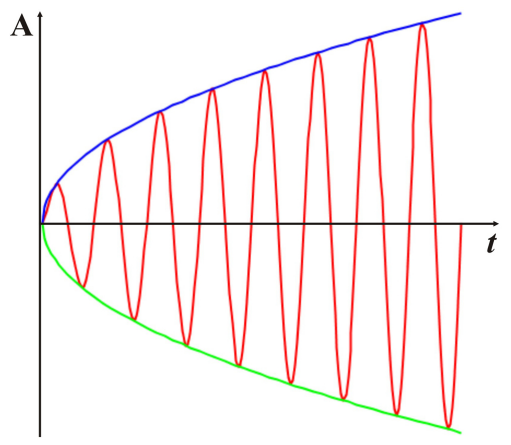

Figura 8: Amplitude da onda sonora durante o crescendo. que permite reconhecermos diferenças quanto à origem de emissão é denominada timbre. Isso acontece porque o som é uma composição de ondas sonoras, denominadas harmônicos, e a presença dos harmônicos e suas intensidades relativas na composição de cada som (de cada onda resultante) é diferente para sons produzidos por fontes diferentes; o som produzido por uma fonte específica carrega uma "assinatura" que o caracteriza como produzido por aquela fonte; o som tem um timbre específico, tem uma "personalidade". Descrever esse padrão significa observar o espectro sonoro da onda produzida 12 .

Para melhor compreender o fenômeno, lembremos que, de acordo com a análise de Fourier, qualquer função periódica, como é a função que representa um som musical, pode ser escrita como a soma de funções senoidais 14, 15], da forma

$$
y=\sum_{i} y_{i}=\sum_{i} A_{i} \sin \left(2 \pi f_{i} t\right)
$$

onde $A_{i}$ e $f_{i}$ representam, respectivamente, a amplitude e a frequência de cada onda individual. Tais ondas senoidais individuais são justamente os harmônicos. Na Figura 9 estão ilustrados os gráficos dos três primeiros harmônicos da nota Sol grave emitida por uma flauta transversal 16]. As curvas vermelha, azul e verde correspondem aos primeiro $(388 \mathrm{~Hz})$, segundo $(776 \mathrm{~Hz})$ e terceiro $(1164 \mathrm{~Hz})$ harmônicos, respectivamente. As constantes $A_{1}, A_{2}$ e $A_{3}$ indicam as amplitudes relativas desses harmônicos em unidades arbitrárias.

Considerando que estes são os harmônicos mais presentes, podemos traçar a onda resultante, como mostra a Figura 10

\section{A pulsação e o rítmo}

Aquele que dedicar um tempo a analisar os gestos de um maestro a reger uma orquestra que apresenta várias peças musicais em sequência perceberá que, para determinados grupos de estilos musicais, há algo de semelhante na forma de mover as mãos, uma forma de marcar a passagem do tempo, de dividi-lo em ciclos e estes em pulsos. Os pulsos representam batidas uniformes, não

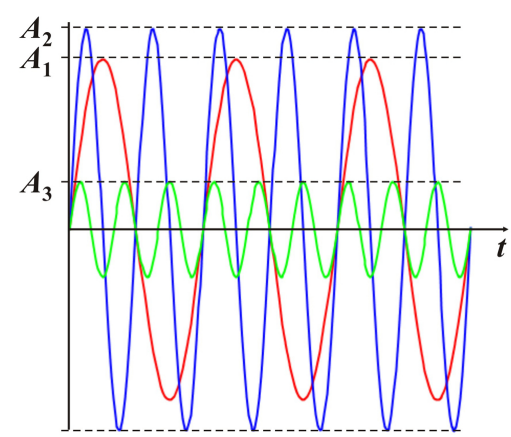

Figura 9: Três primeiros harmônicos da nota Sol da flauta transversal. 


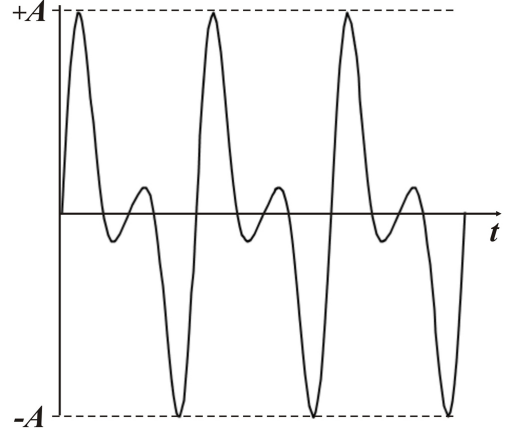

Figura 10: Onda sonora da nota Sol da flauta transversal.

necessariamente emitidas ou ouvidas mas sentidas, que servem para quantizar o texto musical. A sequência periódica dos pulsos é denominada pulsação 7,10 . Quanto maior for o intervalo entre um pulso e o subsequente, mas lenta será a música executada, e vice-versa. A pulsação está, portanto, relacionada também à rapidez com que a música acontece, o que é denominado andamento. Um instrumento cuja finalidade consiste em indicar a pulsação é o metrônomo. Este dispositivo especifica a pulsação via batimentos por minuto (bpm), ou seja, estabelece uma frequência para a pulsação.

Tomando como base uma determinada pulsação, é possível construir diferentes padrões de batidas percussivas característicos de determinados estilos musicais. Um tal padrão é denominado rítmo 11]. Estruturas rítmicas características do baião, do samba e do frevo, por exemplo, embora diferentes, são todas montadas sobre uma pulsação de natureza binária. Isto significa que a estrutura rítmica completa um ciclo em um intervalo temporal determinado por dois pulsos. Seguindo o mesmo pensamento, podemos perceber que um rítmo como o bolero tem uma estrutura montada sobre uma pulsação quaternária, enquando a valsa é estruturada em uma pulsação ternária.

Todas essas informações devem estar presentes na escrita musical. Inicialmente deve ser indicada a natureza do ciclo - se binário, ternário, quaternário. Este ciclo elementar é, portanto, denominado compasso [6,10]. A divisão de cada compasso é feita através de uma barra vertical denominada barra de compasso; o tipo de compasso é estabelecido a partir da indicação de uma fórmula denominada fórmula de compasso, da forma

$$
\frac{b}{a}
$$

A informação aqui presente é que no compasso representado cabem $b$ notas do tipo $1 / a$, sendo esta última a figura de valor fração da nota inteira que denominamos semibreve. Os compassos mais comuns são denominados compassos simples:

- Compasso quaternário: é representado pela fórmula de compasso 4/4, indicando que o intervalo correspondente ao compasso é subdividido em quatro intervalos temporais iguais de duração igual à da figura semínima, ou seja, cabem no compasso 4 notas (ou pausas, ou combinações de notas e pausas) do tipo $1 / 4$. Se a semínima representa a unidade de tempo do compasso, indicando consequentemente a pulsação, podemos afirmar que a frequência de pulsação do compasso quaternário é do tipo $f=4 / T$, onde $T$ representa o período do compasso.

- Compasso binário: indicado pela fórmula de compasso $2 / 4$, tem o período subdividido em dois intervalos temporais iguais de duração igual à da figura semínima. Esta então representa a unidade de tempo do compasso. Podemos definir a frequência de pulsação do compasso binário como sendo $f=2 / T$, seguindo a notação já estabelecida.

- Compasso ternário: é indicado pela fórmula de compasso $3 / 4$, ou seja, cabem no compasso 3 notas (ou pausas, ou combinações de notas e pausas) do tipo 1/4. Se a semínima representa a unidade de tempo do compasso, a pulsação ocorre com uma frequência do tipo $f=3 / T$.

Há ainda os compassos compostos, que podem ser entendidos como resultado de uma subdivisão ternária dos compassos simples. Os mais comuns são:

- Compasso binário 6/8: a fórmula de compasso indica que cabem no compasso 6 notas (ou pausas, ou combinações de notas e pausas) do tipo $1 / 8$, ou seja, o período $T$ do compasso é igualmente dividido em seis intervalos menores de duração igual à da nota colcheia. Note que é um compasso binário; pode ser pensado como dividido em duas pulsações principais. Nesse caso a frequência de pulsação pode ser representada por $f=2 / T$, a mesma do compasso binário simples. Caso se queira entender como representativa da pulsação a colcheia, a frequência de pulsação passa a ser indicada então por $f=6 / T$, de maneira que cada pulsação binária é dividida em três partes.

- Compasso ternário 9/8: no período deste compasso cabem 9 notas do tipo 1/8, ou seja, cabem nove colcheias. A frequência das pulsações principais é do tipo $f=3 / T$. Já a frequência das pulsações resultantes da subdivisão ternária é $f=9 / T$.

- Compasso quaternário 12/8: cabem no período 12 notas do tipo $1 / 8$. A frequência das pulsações principais é dada por $f=4 / T$. A frequência das pulsações em termos das colcheias é, então, $f=12 / T$.

Existem outras combinações possíveis, ou seja, outros tipos de compassos que são montados a partir de diferentes combinações de pulsos, alguns inclusive de difícil compreensão. Limitamo-nos aos mais comumente utilizados. 


\section{A melodia}

A definição de melodia está associada ao fenômeno da combinação de sons de maneira sucessiva, sons com diferentes características que são emitidos em sequência. A sequência de sons de diferentes frequências, ou seja, a sequência de sons de diferentes alturas (onde altura representa a propriedade dos sons serem mais agudos ou mais graves), resulta em uma estrutura que podemos denominar de "desenho melódico" ou melodia 6 .

Nesse contexto é interessante falarmos do conceito de intervalo, representado matematicamente por

$$
N^{\mathrm{a}}=\frac{f_{i+N-1}}{f_{i}}
$$

onde $N^{\text {a }}$ indica o intervalo (de segunda, terça, quarta, etc), $f_{i}$ indica a frequência da nota mais grave e $f_{i+N-1}$ indica a frequência da nota mais aguda, de forma que $f_{i+N-1}>f_{i}$. Dessa forma, um intervalo de quinta, por exemplo, é dado por

$$
5^{\mathrm{a}}=\frac{f_{i+4}}{f_{i}},
$$

onde $i$ indica a posição da nota dentro da escala, como será visto adiante. Esses intervalos ainda podem sofrer variações que lhe permitem ser classificados como justos, maiores, menores, aumentados e diminutos. Passemos então a analisar as escalas musicais mais comuns. Estas são, portanto, estruturas melódicas montadas a partir da combinação de intervalos.

\subsection{A escala maior}

A escala maior, por exemplo, é formada a partir da seguinte estrutura 7]:

$$
\begin{aligned}
& I \underset{2^{\mathrm{a}} \mathrm{M}}{\longrightarrow} I I \underset{2^{\mathrm{a}} \mathrm{M}}{\longrightarrow} I I I \underset{2^{\mathrm{a}} \mathrm{m}}{\longrightarrow} I V \underset{2^{\mathrm{a}} \mathrm{M}}{\longrightarrow} V \underset{2^{\mathrm{a}} \mathrm{M}}{\longrightarrow} \\
& V I \underset{2^{\mathrm{a}} \mathrm{M}}{\longrightarrow} V I I \underset{2^{\mathrm{a} m}}{\longrightarrow} V I I I,
\end{aligned}
$$

onde os numerais romanos indicam a função de cada nota dentro da escala, se tônica (a primeira nota da escala), segunda, terça, etc, e $2^{\mathrm{a}} \mathrm{M}$ e $2^{\mathrm{a}} \mathrm{m}$ indicam intervalos de segunda maior e segunda menor, respectivamente.

O intervalo entre os graus $I$ e $I I$ dessa escala é de segunda maior, o mesmo entre os pares de graus $(I I, I I I)$, $(I V, V),(V, V I)$ e $(V I, V I I)$. As razões entre as frequências dos pares citados são iguais:

$$
2^{\mathrm{a}} \mathrm{M}=\frac{f_{I I}}{f_{I}}=\frac{f_{I I I}}{f_{I I}}=\frac{f_{V}}{f_{I V}}=\frac{f_{V I}}{f_{V}}=\frac{f_{V I I}}{f_{V I}} .
$$

Entre os pares de graus $(I I I, I V)$ e $(V I I, V I I I)$, o intervalo existente é de segunda menor:

$$
2^{\mathrm{a}} \mathrm{m}=\frac{f_{I V}}{f_{I I I}}=\frac{f_{V I I I}}{f_{V I I}} .
$$

Essas duas primeiras classificações, os intervalos de segunda maior e de segunda menor, são também denominados, respectivamente, tons e semitons. Assim sendo, é comum representar a estrutura intervalar que caracteriza a escala maior da forma

$$
\begin{gathered}
I \underset{\text { Tom }}{I} \quad \underset{\text { Tom }}{\longrightarrow} \quad I I I \underset{\text { Semi }}{\longrightarrow} I V \underset{\text { Tom }}{\longrightarrow} V \\
\underset{\text { Tom }}{\longrightarrow} \text { Tom }
\end{gathered}
$$

onde a abreviação 'semi' representa semiton.

Continuando a análise da estrutura da escala, vemos que o intervalo entre os pares de graus $(I, I I I),(I V, V I)$, $(V, V I I)$ é de terça maior:

$$
3^{\mathrm{a}} \mathrm{M}=\frac{f_{I I I}}{f_{I}}=\frac{f_{V I}}{f_{I V}}=\frac{f_{V I I}}{f_{V}} .
$$

$\mathrm{O}$ intervalo existente entre os pares $(I I, I V),(I I I, V)$ e $(V I, V I I I)$ é de terça menor:

$$
3^{\mathrm{a}} \mathrm{m}=\frac{f_{I V}}{f_{I I}}=\frac{f_{V}}{f_{I I I}}=\frac{f_{V I I I}}{f_{V I}} .
$$

O intervalo entre os pares $(I, I V),(I I, V),(I I I, V I)$, $(V, V I I I)$ é de quarta justa:

$$
4^{\mathrm{a}} \mathrm{J}=\frac{f_{I V}}{f_{I}}=\frac{f_{V}}{f_{I I}}=\frac{f_{V I}}{f_{I I I}}=\frac{f_{V I I I}}{f_{V}} .
$$

O intervalo entre os graus $I V$ e $V I I$ é de quarta aumentada:

$$
4^{\mathrm{a}} \mathrm{aum}=\frac{f_{V I I}}{f_{I V}} .
$$

O intervalo entre os pares $(I, V),(I I, V I),(I I I, V I I)$, $(I V, V I I I)$ é de quinta justa:

$$
5^{\mathrm{a}} \mathrm{J}=\frac{f_{V}}{f_{I}}=\frac{f_{V I}}{f_{I I}}=\frac{f_{V I I}}{f_{I I I}}=\frac{f_{V I I I}}{f_{I V}} .
$$

O intervalo entre os pares $(I, V I)$ e $(I I, V I I)$ é de sexta maior:

$$
6^{\mathrm{a}} \mathrm{M}=\frac{f_{V I}}{f_{I}}=\frac{f_{V I I}}{f_{I I}} .
$$

O intervalo entre os graus $I I I$ e VIII é de sexta menor:

$$
6^{\mathrm{a}} \mathrm{m}=\frac{f_{V I I I}}{f_{I I I}} .
$$

O intervalo entre os graus $I$ e VII é de sétima maior:

$$
7^{\mathrm{a}} \mathrm{M}=\frac{f_{V I I}}{f_{I}} .
$$

Já entre $I I$ e VIII é de sétima menor:

$$
7^{\mathrm{a}} \mathrm{m}=\frac{f_{V I I I}}{f_{I I}} .
$$


O intervalo entre os graus $I$ e $V I I I$ é de oitava justa:

$$
8^{\mathrm{a}} \mathrm{J}=\frac{f_{V I I I}}{f_{I}}=2
$$

Com exceção do intervalo de $8^{\mathrm{a}} \mathrm{J}$, em que a frequência do som mais agudo é exatamente o dobro da frequência do som mais grave, não é possível ainda mensurar os valores que representam os intervalos descritos até então. Isso ficará mais claro na próxima subseção.

\subsection{A transposição e a escala cromática}

Antes de passar à análise de outros tipos de escala, convém fazer uma breve discussão sobre o ato da transposição (ou transporte), o que consiste em escrever, ler, cantar ou tocar determinada melodia em uma altura diferente [6]. Tomemos como exemplo a escala de Dó maior. Esta, conhecida também como escala diatônica, segue exatamente a estrutura da Equação (10), conforme ilustra a Figura 11 .

Percebamos, porém, que, utilizando as mesmas notas da escala diatônica, uma escala começando na nota Ré não obedece à estrutura de uma escala maior. Isso só é possível se as frequências dos graus $I I I$ (o Fá) e VII (o Dó) forem alteradas em um valor correspondente a um intervalo de semiton acima, conforme ilustra a Figura 12

A escala de Fá maior, por outro lado, tem uma alteração na frequência do grau $I V$ (o $S i$ ) equivalente a um intervalo de semiton abaixo. Isso está ilustrado na Figura 13

Tais alterações são denominadas acidentes: a alteração "para cima" (o aumento da frequência) é denominada sustenido e é representada pelo símbolo \# colocado ao lado esquerdo da nota grafada no pentagrama; a alteração "para baixo" (a diminuição da frequência) chama-se bemol e é representada por um $b$ ao lado esquerdo da nota grafada no pentagrama. Há ainda situações em que

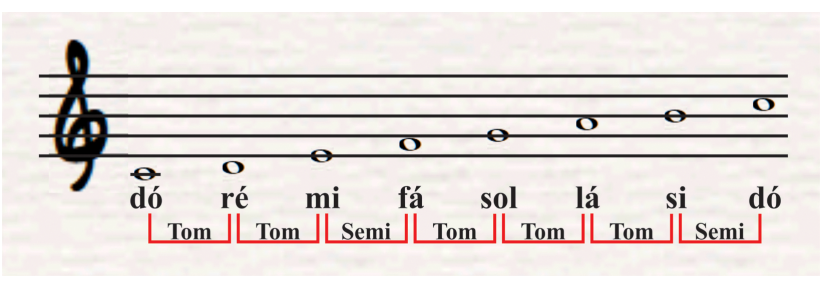

Figura 11: Escala de Dó maior.

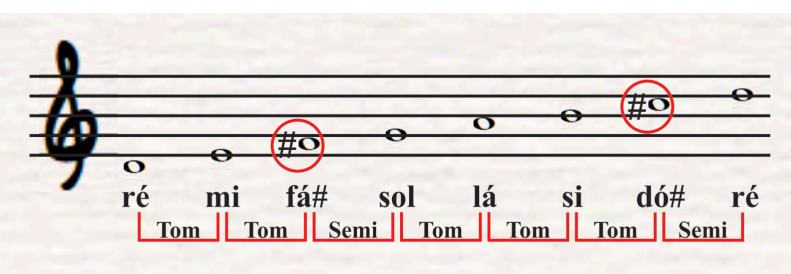

Figura 12: Escala de ré maior.

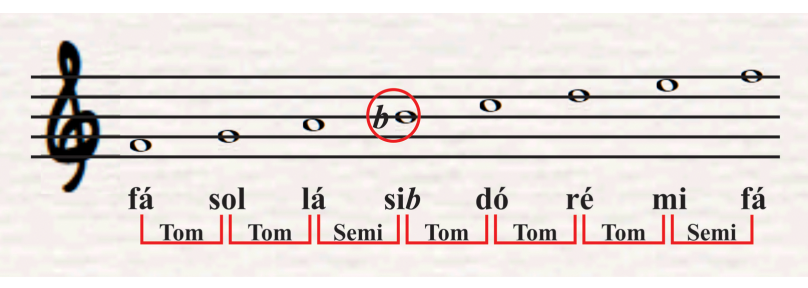

Figura 13: Escala de Fá maior.

a alteração sustenido aplicada a determinada nota indica a mesma frequência que a alteração bemol na nota ascendente vizinha. Como exemplo, as notas Dó\# e Réb, embora diferentes no contexto da escrita musical, de fato são sons de mesma frequência. Dizemos então que tais notas são enarmônicas; o fenônemo recebe o nome de enarmonia.

Vimos, portanto, que os acidentes surgem das necessidades impostas pelo ato da transposição, e que entre as notas naturais (pense nas teclas brancas do piano) surgem notas de frequências intermediárias conhecidas como notas cromáticas (pense nas teclas pretas do piano). Dessa forma, a escala diatônica, antes composta de sete notas, é acrescida de mais cinco notas correspondendo às frequências intermediárias entre os sete tons naturais, com exceção das regiões entre os pares de graus $(I I I, I V)$ e $(V I I, V I I I)$, cujos intervalos já são de semitom. A partir da escala cromática então formada por essas doze notas, também conhecida como escala temperada (ou de igual temperamento), podemos definir o intervalo de semitom 17. Pensemos neste intervalo como o número que, multiplicado pela frequência de uma nota da escala, resulta na frequência da nota imediatamente seguinte. Lembremos ainda que, conforme explicitado na Equação (20), o intervalo de oitava justa é resultado do produto da frequência de uma determinada nota por um fator 2. O fator que representa o intervalo de semitom deve ser, então, o número $s$ que, participando do processo de multiplicação de uma frequência para obtenção da seguinte doze vezes sucessivas, resulte no fator 2 , ou seja,

$$
\begin{gathered}
s^{12}=2, \\
s=\sqrt[12]{2} \approx 1,05946 .
\end{gathered}
$$

Agora temos condições de pensar numericamente os intervalos vistos anteriormente. O intervalo de tom, por exemplo, corresponde a uma razão de frequências igual a $s^{2}$, ou seja, compreende dois semitons. Dessa forma, as frequências dos graus $I$ e $I I$ da escala maior, o mesmo valendo para os demais pares de graus cujos intervalos sejam de tom, ou seja, de $2^{\mathrm{a}} \mathrm{M}$, relacionam-se como segue:

$$
f_{I I}=s^{2} f_{I}
$$

Ainda como exemplo, notemos que o intervalo de quarta justa compreende dois tons e um semitom, portanto cinco semitons; a razão entre as frequências é igual 
a $s^{5}$. A relação entre as frequências dos graus $I I$ e $V$ da escala maior, portanto, assim como para os demais pares de graus cujos intervalos sejam de $4^{\mathrm{a}} \mathrm{J}$, é da forma:

$$
f_{V}=s^{5} f_{I I} \text {. }
$$

Seguindo o mesmo procedimento, é possível obter os valores das razões entre as frequências de todos os demais intervalos conhecidos, sendo desnecessário aqui repetir o processo para todos eles. Convém, porém, explicitar as relações de frequências resultantes das alterações sustenido e bemol discutidas anteriormente. Seja $f_{\text {nota\# }}$ frequência da nota alterada pelo sustenido e seja $f_{\text {nota }}$ a frequência da nota natural, a relação entre tais frequências é

$$
f_{\text {nota\# }}=s f_{\text {nota }} .
$$

Já a relação entre a frequência da nota alterada pelo bemol $f_{\text {notab }}$ e $f_{\text {nota }}$ é

$$
f_{\text {notab }}=\frac{f_{\text {nota }}}{s} .
$$

Dessa forma, temos que $f_{\mathrm{Fá \#}}=s f_{\mathrm{Fá}}$ e $f_{\mathrm{Si} b}=f_{\mathrm{Si}} / s$, por exemplo. Podemos ainda observar que o fenômeno da enarmonia é consequência do igual temperamento da escala cromática. Consideremos, portanto, $f_{\mathrm{n}}$ como sendo a frequência de uma nota qualquer e $f_{\mathrm{n}+1}$ como sendo a frequência da nota que forma com a anterior um intervalo de um tom inteiro. Temos que

$$
f_{(\mathrm{n}+1) b}=\frac{f_{(\mathrm{n}+1)}}{s}=\frac{s^{2} f_{\mathrm{n}}}{s}=s f_{\mathrm{n}}=f_{(\mathrm{n}) \#} .
$$

\subsection{A escala menor}

Tomando como base a escala maior, podemos montar uma escala relativa, que denominamos escala menor. Admitindo o sexto grau da escala maior como sendo o primeiro grau dessa nova escala, tendo o cuidado de manter a ordem cíclica, ou seja, entendendo que depois do sétimo grau da escala maior voltamos ao primeiro grau, este representa na escala relativa o terceiro grau. Dessa forma, a escala menor tem a seguinte estrutura:

$$
\begin{aligned}
& I \underset{2^{\mathrm{a} M}}{\longrightarrow} I I \underset{2^{\mathrm{a}} \mathrm{m}}{\longrightarrow} I I I \underset{2^{\mathrm{a}} \mathrm{M}}{\longrightarrow} I V \underset{2^{\mathrm{a}} \mathrm{M}}{\longrightarrow} V \\
& \underset{2^{\mathrm{a} m}}{\longrightarrow} V I \underset{2^{\mathrm{a}} \mathrm{M}}{\longrightarrow} V I I \underset{2^{\mathrm{a}} \mathrm{M}}{\longrightarrow} V I I I .
\end{aligned}
$$

Pensando em termos de tons e semitons, a estrutura da escala menor tem a forma

$$
\begin{aligned}
& I \underset{\text { Tom }}{\longrightarrow} I I \underset{\text { Semi }}{\longrightarrow} I I I \underset{\text { Tom }}{\longrightarrow} I V \underset{\text { Tom }}{\longrightarrow} V \\
& \underset{\text { Semi }}{\longrightarrow} \text { Tom }
\end{aligned}
$$

A Figura 14 ilustra a escala de Lá menor.

Uma análise cuidadosa, da forma feita para o caso da escala maior, permite observar que toda a estrutura intervalar foi modificada. Por exempo, os intervalos de terça menor agora correspondem a outros pares de graus:

$$
3^{\mathrm{a}} \mathrm{m}=\frac{f_{I I I}}{f_{I}}=\frac{f_{I V}}{f_{I I}}=\frac{f_{V I}}{f_{I V}}=\frac{f_{V I I}}{f_{V}} .
$$

$\mathrm{E}$ isso pode ser observado para os demais intervalos. Entre os graus $I I$ e $V I$, porém, surge um intervalo que não havia sido observado na escala maior (pelo menos não dentro de uma única oitava) - o de quinta diminuta:

$$
5^{\mathrm{a}} \operatorname{dim}=\frac{f_{V I}}{f_{I I}}=s^{6} .
$$

Na escala de Lá menor da Figura 14, podemos ver que este intervalo existe entre as notas Si e Fá, compreendento três tons, quando somados os intervalos sucessivos entre as cinco notas. Extrapolando a oitava atual, essa mesma distância intervalar pode ser vista também entre as notas $F a ́$ e $S i$ (note que agora estamos considerando o $S i$ com uma frequência que é o dobro da nota $S i$ considerada anteriormente). Mas agora o intervalo como um todo compreente quatro notas; é então o intervalo de quarta aumentada já tratado aqui, desta vez escrito como a razão entre as frequências do sexto e do nono graus:

$$
4^{\mathrm{a}} \mathrm{aum}=\frac{f_{I X}}{f_{V I}}=s^{6} .
$$

Do ponto de vista prático, portanto, os intervalos de quarta aumentada e quinta diminuta são iguais.

\subsection{A escala menor harmônica}

A escala menor harmônica é uma variação da escala menor natural [11]. É obtida quando a frequência do sétimo grau é aumentada em um semiton, ou seja, quando é multiplicada pelo fator $s$. Vejamos a estrutura:

$$
\begin{aligned}
& I \underset{2^{\mathrm{a}} \mathrm{M}}{\longrightarrow} I I \underset{2^{\mathrm{a}} \mathrm{m}}{\longrightarrow} I I I \underset{{ }^{\mathrm{a}} \mathrm{M}}{\longrightarrow} I V \underset{2^{\mathrm{a}} \mathrm{M}}{\longrightarrow} V \\
& \underset{2^{\mathrm{a} m}}{\longrightarrow} V I \underset{3^{\mathrm{a} m}}{\longrightarrow} V I I \underset{2^{\mathrm{a}} \mathrm{m}}{\longrightarrow} V I I I .
\end{aligned}
$$

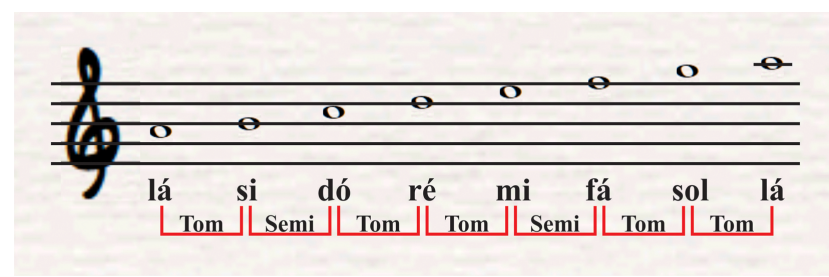

Figura 14: Escala de Lá menor. 
Em termos de tons e semitons, temos

$$
\begin{aligned}
& I \underset{\text { Tom }}{\longrightarrow} I I \underset{\text { Semi }}{\longrightarrow} I I I \underset{\text { Tom }}{\longrightarrow} I V \underset{\text { Tom }}{\longrightarrow} V \\
& \underset{\text { Semi }}{\longrightarrow} \quad V I \underset{\mathrm{T}+\mathrm{S}}{\longrightarrow} V I I \underset{\mathrm{Semi}}{\longrightarrow} V I I I,
\end{aligned}
$$

onde $T+S$ é uma abreviação de tom + semiton, significando que entre os graus $V I$ e $V I I$ o intervalo foi aumentado para uma $3^{a} m$ - correspondente a um tom $e$ meio, ou seja, $f_{V I I} / f_{V I}=s^{3}$, e o intervalo entre os graus $V I I$ e $V I I I$ foi diminuído para uma $2^{a} m$ - um semiton. Surge também, entre os graus $I I I$ e VII, um intervalo ainda não mencionado - o de quinta aumentada:

$$
5^{\mathrm{a}} \mathrm{aum}=\frac{f_{V I I}}{f_{I I I}}=s^{8} .
$$

As alterações podem ser melhor observadas na Figura 15. onde está ilustrada a escala de Lá menor harmônica. $\mathrm{O}$ intervalo de quinta aumentada, equivalente a quatro tons inteiros, existe entre as notas Dó e Sol\#. Um intervalo também de quatro tons inteiros resulta da soma dos intervalos sucessivos entre as notas $L a ́$ e $F a ́$, mas desta vez compreendidos entre seis notas. A denominação neste caso é de sexta menor.

\subsection{A escala menor melódica}

A escala menor melódica é obtida também a partir de alterações na escala menor natural; desta vez, os graus $V I$ e $V I I$ são ambos modificados por um fator $s$ - as frequências correspondentes são aumentadas em um semiton para cada nota [11]. A estrutura é da forma

$$
\begin{aligned}
& I \underset{2^{\mathrm{a}} \mathrm{M}}{\longrightarrow} I I \underset{2^{\mathrm{a} m}}{\longrightarrow} I I I \underset{2^{\mathrm{a}} \mathrm{M}}{\longrightarrow} I V \underset{2^{\mathrm{a}} \mathrm{M}}{\longrightarrow} V \\
& \underset{2^{\mathrm{a}} \mathrm{M}}{\longrightarrow} V I \underset{2^{\mathrm{a}} \mathrm{M}}{\longrightarrow} V I I \underset{2^{\mathrm{a} m}}{\longrightarrow} V I I I .
\end{aligned}
$$

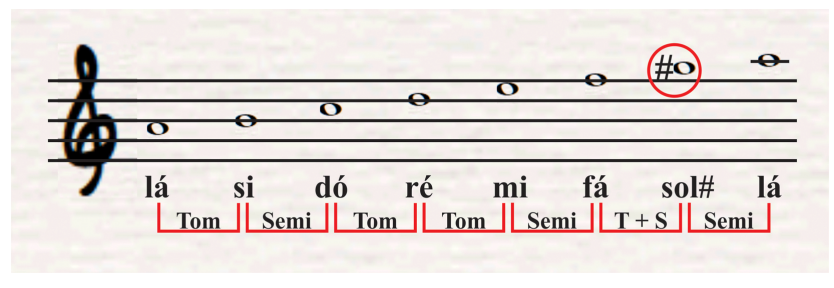

Figura 15: Escala de Lá menor harmônica.
Em termos de tons e semitons, temos

$$
\begin{aligned}
& I \underset{\text { Tom }}{\longrightarrow} I I \underset{\text { Semi }}{\longrightarrow} I I I \underset{\text { Tom }}{\longrightarrow} I V \underset{\text { Tom }}{\longrightarrow} V \\
& \underset{\text { Tom }}{\longrightarrow} \text { Tom } \\
& \underset{\text { Tomi }}{\longrightarrow} V I I \underset{\text { Semi }}{\longrightarrow} V I I I .
\end{aligned}
$$

Conforme pode ser visto, com exceção dos intervalos entre os pares de graus $(I I, I I I)$ e $(I I I, I V)$, o restante da estrutura da escala menor melódica é igual à da escala maior. A Figura 16 traz o exemplo da escala de Lá menor melódica.

Há diversas outras escalas, das mais diferentes naturezas, mas acreditamos que o roteiro para análise das estruturas intervalaves já está bem estabelecido. Temos falado até então dos intervalos classificados na literatura musical como intervalos melódicos, tendo em vista que estamos tratando de sons que são emitidos/tocados de maneira sucessiva. Na seção seguinte passamos a falar de estruturas que são montadas a partir de intervalos harmônicos que, embora as classificações representem as mesmas razões de frequências discutidas até agora, tratam de sons que são emitidos/tocados simultaneamente, portanto de ondas sonoras que estão presentes simultaneamente em um mesmo ponto do meio nos instantes considerados.

\section{A harmonia}

A harmonia estuda as diversas combinações de sons emitidos simultaneamente. Trata de como são constituídos os acordes, das consonâncias e dissonâncias e de como os acordes se organizam para o acompanhamento, ou seja, para a harmonização de melodias [18]. Nesta seção, tratamos apenas do entendimento físico das combinações de sons na formação dos acordes mais comuns.

Um grupo de no mínimo três notas diferentes, quando tocadas simultaneamente, forma uma estrutura harmônica denominada de acorde. O fenômeno físico que ocorre é um somatório de ondas, cuja configuração resultante pode ser entendida assumindo-se a validade do princípio da superposição, cujo enunciado afirma que as amplitudes das ondas se somam algebricamente em cada ponto da região onde se interceptam [15].

Os acordes formados por três notas são os mais simples e são denominados tríades; aqueles formados por qua-

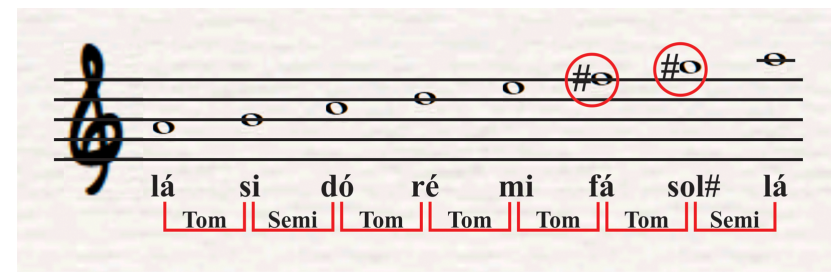

Figura 16: Escala de Lá menor melódica. 
tro notas são denominados tétrades [19]. É importante lembrar que uma onda representativa de uma nota já é uma composição de vários harmônicos que caracterizam a fonte de emissão, mas a nota é identificada pela frequência fundamental. A onda resultante da combinação de uma tríade é dada por

$$
\begin{aligned}
y_{\text {tríade }}= & y_{1}+y_{3}+y_{5} \\
= & \sum_{i} A_{i}^{(1)} \sin \left(2 \pi f_{i}^{(1)} t\right) \\
& +\sum_{j} A_{j}^{(3)} \sin \left(2 \pi f_{j}^{(3)} t\right) \\
& +\sum_{k} A_{k}^{(5)} \sin \left(2 \pi f_{k}^{(5)} t\right),
\end{aligned}
$$

onde $f_{i}^{(1)}, f_{j}^{(3)}$ e $f_{k}^{(5)}$ são, respectivamente, as frequências dos $i$-ésimo, $j$-ésimo e $k$-ésimo harmônicos presentes nas ondas $y_{1}, y_{3}$ e $y_{5}$, formadoras do acorde. Usamos parênteses nos índices sobrescritos para distingui-los de expoentes; neste caso, eles não representam potências. A combinação da tétrade tem uma nota a mais:

$$
\begin{aligned}
y_{\text {tétrade }}= & y_{1}+y_{3}+y_{5}+y_{7} \\
= & \sum_{i} A_{i}^{(1)} \sin \left(2 \pi f_{i}^{(1)} t\right) \\
& +\sum_{j} A_{j}^{(3)} \sin \left(2 \pi f_{j}^{(3)} t\right) \\
& +\sum_{k} A_{k}^{(5)} \sin \left(2 \pi f_{k}^{(5)} t\right) \\
& +\sum_{l} A_{l}^{(7)} \sin \left(2 \pi f_{l}^{(7)} t\right),
\end{aligned}
$$

onde $f_{l}^{(7)}$ é a frequência do $l$-ésimo harmônico presente na nota formada pela onda $y_{7}$. A nota $y_{1}$ recebe o nome de tônica ou fundamental; é a nota que dá nome ao acorde e será indicada aqui por $T$. As demais notas, $y_{3}, y_{5}$ e $y_{7}$ são, respectivamente, a terça, a quinta e a sétima do acorde, obedecendo as relações intervalares discutidas na seção anterior.

As tríades podem ser classificadas como maiores, menores, aumentadas, diminutas. As tríades maiores têm a seguinte estrutura:

$$
T \underbrace{+}_{3^{\mathrm{a} M}} 3^{\mathrm{a}} \mathrm{M} \underbrace{+}_{3^{\mathrm{a}} \mathrm{m}} 5^{\mathrm{a}} \mathrm{J}
$$

Para este caso, as frequências dos primeiros harmônicos de cada nota relacionam-se da seguinte forma:

$$
f_{1}^{(3)}=s^{4} f_{1}^{(1)} \quad \text { e } \quad f_{1}^{(5)}=s^{3} f_{1}^{(3)}=s^{7} f_{1}^{(1)} .
$$

A Figura 17 mostra a representação no pentagrama do acorde de Dó maior. Da esquerda para a direita, os dois primeiros acordes grafados obedecem à estrutura apresentada na Equação (34) e as frequências dos primeiros harmônicos de cada nota estão relacionadas conforme

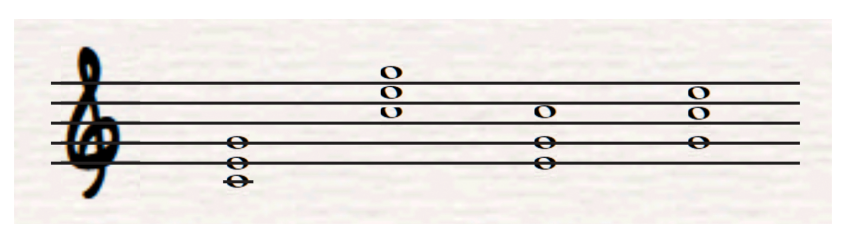

Figura 17: Acorde de Dó maior.

a Equação (35). Em ordem crescente de frequência, as notas são Dó, $M i$ e Sol. O que difere uma formação da outra é que o segundo acorde está em uma oitava acima com relação ao primeiro, ou seja, as notas que estruturam o segundo acorde têm frequências que são o dobro das frequências das notas correspondentes do primeiro acorde. Nesta forma de estruturar, a fundamental é a nota mais grave (de menor frequência) do acorde. Uma tríade deste tipo é dita estar em sua posição fundamental [19]. Uma tríade cuja $3^{\text {a }}$ seja a nota mais grave é dita estar na primeira inversão - é o caso do terceiro acorde. O quarto acorde está estruturado de maneira que a $5^{\mathrm{a}}$ é a nota mais grave. Neste caso, a tríade é dita estar na segunda inversão.

Podemos notar que, quando invertidas as tríades, as relações intervalares são alteradas; a estrutura (34) é modificada, assim como as Equações (35). Na primeira inversão, a relação entre as frequências dos primeiros harmônicos da nota mais grave (que agora é a $3^{\mathrm{a}}$ ) e da fundamental corresponde a um intervalo de $6^{\mathrm{a}} \mathrm{m}$. Entre a $5^{\mathrm{a}}$ e a fundamental, o invervalo é de $4^{\mathrm{a}} \mathrm{J}$.

As tríades menores têm a seguinte estrutura:

$$
T \underbrace{+}_{3^{\mathrm{a}} \mathrm{m}} 3^{\mathrm{a} m} \underbrace{+}_{3^{\mathrm{a}} \mathrm{M}} 5^{\mathrm{a}} \mathrm{J}
$$

As frequências dos primeiros harmônicos de cada nota obedecem às relações

$$
f_{1}^{(3)}=s^{3} f_{1}^{(1)} \quad \text { e } \quad f_{1}^{(5)}=s^{4} f_{1}^{(3)}=s^{7} f_{1}^{(1)} .
$$

A Figura 18 ilustra o acorde de Dó menor na posição fundamental, na posição fundamental uma oitava acima, na primeira inversão e na segunda inversão, respectivamente, da esquerda para a direita.

$\mathrm{O}$ que diferencia basicamente uma tríade maior de uma tríade menor é a nota que representa a $3^{\text {a }}$ do acorde. A $5^{\mathrm{a}}$ é a mesma nos dois casos. Quando há alteração nas frequências da $5^{\mathrm{a}}$, as tríades passam a ser aumentadas

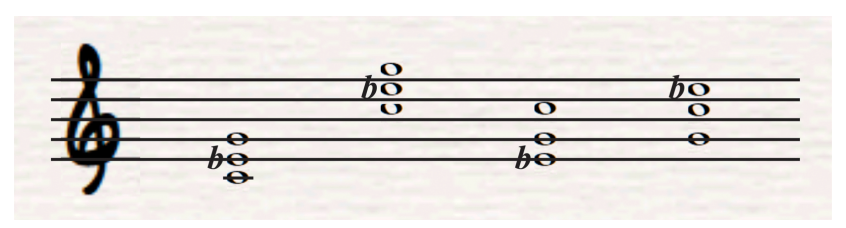

Figura 18: Acorde de Dó menor. 
ou diminutas. A estrutura das tríades aumentadas é a seguinte:

$$
T \underbrace{+}_{3^{\mathrm{a}} \mathrm{M}} 3^{\mathrm{a}} \mathrm{M} \underbrace{+}_{3^{\mathrm{a}} \mathrm{M}} 5^{\mathrm{a}} \text { aum. }
$$

As frequências que compoem a $5^{\mathrm{a}}$ da tríade aumentada são obtidas multiplicando-se as frequências que compoem a $5^{\mathrm{a}}$ da tríade maior por um fator $s$. A estrutura das tríades diminutas, por outro lado, é

$$
T \underbrace{+}_{3^{\mathrm{a} m}} 3^{\mathrm{a} m} \underbrace{+}_{3^{\mathrm{a}} \mathrm{m}} 5^{\mathrm{a}} \operatorname{dim} \text {. }
$$

Neste caso, as frequências que compoem a $5^{\mathrm{a}}$ da tríade diminuta são obtidas multiplicando-se as frequências que compoem a $5^{\mathrm{a}}$ da tríade menor por um fator $1 / \mathrm{s}$. A Figura 19 ilustra, no pentagrama, as tríades aumentada e diminuta de Dó, respectivamente.

Passemos a fazer uma breve discussão sobre as tétrades - os acordes de sétima 11]. Quando acrescentamos uma sétima maior a uma tríade maior, obtemos o acorde de sétima maior, cuja estrutura é

$$
T \underbrace{+}_{3^{\mathrm{a}} \mathrm{M}} 3^{\mathrm{a}} \mathrm{M} \underbrace{+}_{3^{\mathrm{a}} \mathrm{m}} 5^{\mathrm{a}} \mathrm{J} \underbrace{+}_{3^{\mathrm{a}} \mathrm{M}} 7^{\mathrm{a}} \mathrm{M} .
$$

As relações entre as frequências dos primeiros harmônicos das notas formadoras desse acorde são obtidas multiplicando-se $f_{1}^{(5)}$ da Equação 35 por um intervalo de $3^{\mathrm{a}} \mathrm{M}$, como segue:

$$
f_{1}^{(7)}=s^{4} f_{1}^{(5)}=s^{7} f_{1}^{(3)}=s^{11} f_{1}^{(1)} .
$$

Acrescentando-se uma sétima menor a uma tríade maior, obtemos o acorde de sétima ou sétima dominante. A estrutura é a seguinte:

$$
T \underbrace{+}_{3^{\mathrm{a}} \mathrm{M}} 3^{\mathrm{a}} \mathrm{M} \underbrace{+}_{3^{\mathrm{a}} \mathrm{m}} 5^{\mathrm{a}} \mathrm{J} \underbrace{+}_{3^{\mathrm{a}} \mathrm{m}} 7^{\mathrm{a}} \mathrm{m} .
$$

As relações entre frequências são, portanto,

$$
f_{1}^{(7)}=s^{3} f_{1}^{(5)}=s^{6} f_{1}^{(3)}=s^{10} f_{1}^{(1)} .
$$

Uma tríade menor acrescida de uma sétima menor torna-se um acorde menor com sétima, cuja estrutura é

$$
T \underbrace{+}_{3^{\mathrm{a}} \mathrm{m}} 3^{\mathrm{a}} \mathrm{m} \underbrace{+}_{3^{\mathrm{a}} \mathrm{M}} 5^{\mathrm{a}} \mathrm{J} \underbrace{+}_{3^{\mathrm{a}} \mathrm{m}} 7^{\mathrm{a}} \mathrm{m} .
$$

As frequências dos primeiros harmônicos de cada nota se relacionam da forma

$$
f_{1}^{(7)}=s^{3} f_{1}^{(5)}=s^{7} f_{1}^{(3)}=s^{10} f_{1}^{(1)} .
$$

Acordes diminutos ou de sétima diminuta são obtidos acrescentando-se uma sétima diminuta a uma tríade diminuta, conforme a estrutura

$$
T \underbrace{+}_{3^{\mathrm{a} m}} 3^{\mathrm{a} m} \underbrace{+}_{3^{\mathrm{a} m}} 5^{\mathrm{a}} \operatorname{dim} \underbrace{++}_{3^{\mathrm{a} m}} 7^{\mathrm{a}} \operatorname{dim} .
$$

As frequências dos primeiros harmônicos de cada nota se relacionam da forma

$$
f^{(7)}=s^{3} f_{1}^{(5)}=s^{6} f_{1}^{(3)}=s^{9} f_{1}^{(1)} .
$$

Há ainda outras formações possíveis de acordes de sétima. Não é, porém, objetivo nosso tratar de todas as possibilidades. Para melhor visualização das tétrades aqui tratadas, apresentamos, no pentagrama da Figura 20. os acordes de sétima maior, de sétima dominante, menor com sétima e de sétima diminuta de Dó, respectivamente.

Podemos perceber a presença de dois bemois simultâneos na nota $S i$ do último acorde. Esta alteração é chamada de dobrado bemol. Neste caso, a frequência da nota alterada é multiplicada por um fator $1 / s^{2}$. A nota $S i$ alterada dessa forma é, portanto, enarmônica da nota Lá. A alteração no sentido inverso é denominada dobrado sustenido, sendo então a nota alterada multiplicada por um fator $s^{2}$.

\section{Comentários finais}

Temos apresentado algumas relações entre as representações física e musical de alguns fenômenos sonoros importantes para a prática musical. Iniciamos por traçar um paralelo entre a forma de representar o som emitido, dos pontos de vista da Música e da Física, utilizando as respectivas linguagens e considerando as diversas características sonoras tais como duração, altura, timbre e intensidade. Chegamos ao conceito de nota musical e entendemos a simbologia necessária para sua representação. Estudamos as estruturas temporais formadoras do rítmo. Tratamos das relações de frequências presentes nas definições de intervalos musicais e analisamos algumas das escalas musicais mais comuns. Encerramos por analisar algumas das possíveis combinações simultâneas de ondas sonoras, objeto de estudo do campo da harmonia. Os fenômenos físicos, também musicais, cujas representações são aqui estudadas são uma parcela muito pequena do universo de fenômenos que precisam ser entendidos dentro das duas áreas citadas. O entendimento elementar

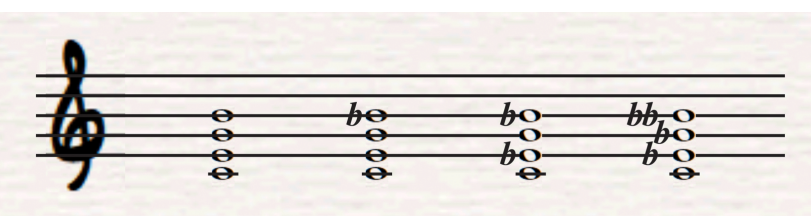

Figura 20: Tétrades de Dó.

Figura 19: Tríades aumentada e diminuta de Dó. 
aqui buscado é, porém, a base de todas as situações mais complexas.

Há vários trabalhos importantes que tratam da relação entre Física e Música. Na referência [20], por exemplo, pode-se encontrar um estudo cuidadoso das condições físicas e matemáticas da consonância de ondas sonoras. Uma análise de uma possível modificação das relações de frequências estabelecidas no temperamento igual pode ser vista em 17]. Outros autores tratam dos princípios físicos envolvidos na produção, propagação e combinação de ondas sonoras em instrumentos musicais de diferentes naturezas, como, por exemplo, em 21 23. Em todos esses trabalhos, é difícil, ou talvez inviável, tratar das relações de frequências entre as ondas sonoras e descrever os fenômenos físicos ocorridos sem utilizar o básico da notação musical, mesmo que não seja este o objetivo. O nosso trabalho, no entanto, teve como objetivo principal tratar da notação musical e de elementos básicos da teoria musical, evidenciar os fenômenos físicos representados por tal notação, com o olhar próprio da Física, considerada como forma equivalente de representar os mesmos fenômenos.

\section{Referências}

[1] F.J. Keller, W.E. Gettys e M.J. Skove, Física (Pearson Education do Brasil, São Paulo, 1999), v. 2.

[2] H.M. Nussenzveig, Curso de Física Básica (Blucher, São Paulo, 2002), v. 2, $4^{\mathrm{a}}$ ed.

[3] H.D. Young e R.A. Freedman, Física II: Termodinâmica e Ondas (Addison Wesley, São Paulo, 2008), $12^{\mathrm{a}}$ ed.

[4] D.R. Raichel, The Science and Applications of Acoustics (Springer Science Business Media Inc., New York, 2006).

[5] J. Fauvel, R. Flood e R. Wilson, Music and Mathematics: From Pythagoras to Fractals (Oxford University Press Inc, New York, 2003).

[6] B. Med, Teoria da Música (Musimed, Brasília, 1996), $4^{\mathrm{a}}$ ed.

[7] J. Harnum, Basic Music Theory: How to Read, Write and Understand Written Music (Sol-Ut Press, Chicago, 2001).

[8] P. Hindemith, Treinamento Elementar para Músicos (Ricordi Brasileira S.A., São Paulo, 1970).

[9] H.R. Strayer, Musical Offerings 4, 1 (2013).

[10] B. Benward e M. Saker, Music in theory and pratice (McGraw-Hill, New York, 2008), $8^{\text {a }}$ ed.

[11] M. Pilhofer e H. Day, Music Theory for Dummies (Wiley Publishing Inc., Indianapolis, 2007).

[12] W.F. Rogers, Physics of Music: Science and Art (Westmont College, Santa Barbara, 2013).

[13] P. Bona, Método Completo para Divisão (Irmãos Vitale, Rio de Janeiro, 1996).

[14] R. Snieder, A Guided Tour of Mathematical Physics (Samizdat Press, Utrecht, 1994).

[15] D. Halliday, R. Resnick e J. Walker, Fundamentos de Física 2: Gravitação, Ondas e Termodinâmica (LTC Editora, Rio de Janeiro, 2009), $8^{\text {a }}$ ed.

[16] S.A. Nascimento, J.D. Dantas, P.C. Souza Segundo e C.A.S. Santos, Rev. Bras. Ens. Fís. 37, 2305 (2015).

[17] H. Hinrichsen, Rev. Bras. Ens. Fís. 38, 1310 (2016).
[18] A. Schoenberg, Structural Functions of Harmony (Faber and Faber, London, 1983), $2^{\mathrm{a}}$ ed.

[19] W. Piston, Harmony (Victor Gollancz, London, 1959).

[20] M. Goto, Rev. Bras. Ens. Fís. 31, 2307 (2009).

[21] J.P. Donoso, A. Tannús, F. Guimarães e T.C. Freitas, Rev. Bras. Ens. Fís. 30, 2305 (2008).

[22] A. Kandus, F.W. Gutmann, C.M.C. Castilho, Rev. Bras. Ens. Fís. 28, 427 (2006).

[23] B.L. Lago, Rev. Bras. Ens. Fís. 37, 1504 (2015). 